\title{
The Study of X-Ray Fluorescence Spectrum of Potsherd in New Stone Age in Zhaotong
}

\author{
Kunli Fu \\ Physics Department, Zhaotong Teacher's College \\ Huancheng East Road, Yunnan 657000, China \\ E-mail: 8896356fkl@163.com \\ Changfen Ding \\ Bureau of Cultural Relics of Zhaotong City \\ Yunnan 657000, China \\ Qiao Lu \& Delan Wu \\ Physics Department, Zhaotong Teacher's College \\ Yunnan 657000, China \\ Bocheng $\mathrm{He}$ \\ The First Middle School of Zhaoyang \\ Yunnan 657000, China
}

\begin{abstract}
Tested the potsherd of new stones age unearthed in Yeshi Mountain of Ludian county, Zhaotong city, Yunnan province with X-ray fluorescence spectrum instrument of automatic scanning in sequence ZSX100e style made in Japanese Science company, analyzed the tested data with SQX normalization software of instrument. Provided charts and datum of another method to test scientifically the sample unearthed in the same place(could be mutual reference to relative datum that result from measurement with X-ray diffraction spectrum instrument, so as to avoid the loss from different measuring method), it's helpful for researcher to analyze from multy-angle. Asertain further the material composition, definite productive place of ancient potsherd. Studied the reason why the shape and structure of them are so similar with that of pottery unearthed in Sanxingdui, tried to explore the internal relation between the ancient culture of Zhaotong, Yunnan and that of Sanxingdui. Taking datum with multiple measuring methods prepared for setting up database of cultural relics unearthed in Zhaotong.
\end{abstract}

Keywords: X-ray, Fluorescence spectrum, Zhaotong, New Stone Age, Potsherd

Lying where Yunnan, Guizhou, Sichuan meet, Zhaotong was once the important Yunnan's gateway to Central Plains, passageway for the culture of Central Plains to spread to Yunnan and communication hub of the famous southern Silk Road. It's one of three early birthplace of culture of Yunnan. The collection of unearthed cultural relics of Zhaotong is the largest among all the cities of Yunnan (nearly 17000 pieces). There are many kinds of cultural relics, ancient remains widely distributed over Zhaotong, such as, stone axe, bone pieces in New Stone Age, red copper, bronze, iron, ceramics in Qing, Han, Tang, Song, Yuan, Ming, Qing Dynasty, the historical remains can be seen everywhere. The birdhead-like pottery, small bottle, drinking vessel in New Stone Age unearthed in Zhaotong are very similar with pottery unearthed in Sanxingdui.

Test and analyze the composition of ancient pottery unearthed in Zhaotong with X-ray fluorescence spectrum, ascertain further other material composition and productive place of them, research its relation with culture of Sanxingdui. The scientific quantitative test of composition of cultural relics unearthed in Zhaotong is very important for setting up database of unearthed cultural relics of Zhaotong to research them deeply.

$\mathrm{X}$-ray fluorescence spectrum analysis is a method to excite the atom of tested matter with X-ray photon of original level 
or other micro particle to emit fluorescence ( X-ray of secondary level) to analyze material composition and chemical state. In modern normal analysis, it's an important way to analyze material composition and has a number of unique merits. First, compared with original level X-ray spectrum analysis, it hasn't continuous X-ray spectrum, the intensity of the background mainly consisted of scattered rays is weak, therefore, the contrast between the spectrum peak and background, and analysis sensitivity get obviously improved. The sample isn't damaged in the testing process. Its measurement of intensity can reappear well, and it's convenient for analysis without damage.

\section{Sample and experimental method}

The studied samples are earth pottery, sand-taken pottery unearthed in the field(about $400 \mathrm{~m}^{2}$ )of Yeshi Mountain in 2002 , by archaeology research institute of Yunnan. The colors of samples are yellow, grey and black. Samples are potsherd of bottom of small flat-bottomed pot and jar, as is shown in Fig. 1.

The testing equipment is X-ray fluorescence spectrum instrument, wavelength chromatic dispersion style of ZSX100e, X-ray generator of high stability, $60 \mathrm{~kW}, 150 \mathrm{~mA}$, the maximum power: $4 \mathrm{~kW}$, Rhodium target X-ray tube of end window type (super thin: $30 \mathrm{~cm}$ ), X-ray systematical spectrum of up irradiation style, the precision of angle-measuring instrument: 2/10000, run automatically. Sample-making equipment: grinder of vibration style made in Japanese Science Company, tablet press: $30 \mathrm{t} / \mathrm{cm}^{2}$, Muffle furnace: $1200^{\circ} \mathrm{C}$.

The quantitative analysis of X-ray fluorescence spectrum means that first, turn the measured characteristic X-ray fluorescence intensity into quality density of tested matter, then get exact quantitative analysis result.

\section{Experimental result and analysis}

The experimental result is in Fig. 2 Fig.4. The experimental data is in Fig. 5.

The experiment start the scientific determination of cultural relics of Zhaotong, we will further research cultural relics of metal, ancient murals material, ancient porcelain, etc. The database of cultural relics of this area will be set up as early as possible.

\section{References}

Arai T, Omote K. (1988). Adv. X-Ray Anal., 1988, 31: 507 514.

Bao S X. (1997). X-Ray Spectrom, 1997, 26: 23 26.

JI Ang, TAO Guang-yi, ZHUO Shang-jun, et al. (2003). X-ray Fluorescence Spectrum Analysis[M]. Beijing: Science Press, 2003.

Omote K, Arai T. (1989). Adv. . X-Ray Anal., 1989, 32: 83 87.

Rousseau R M, Boivin J A. (1998). The Rigaku Jounal. 1998, 15:13 27.
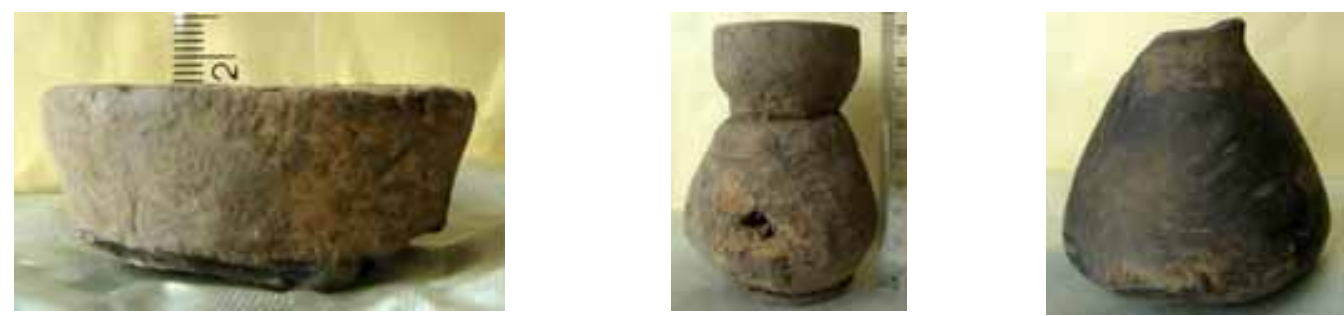

Figure 1. The simples

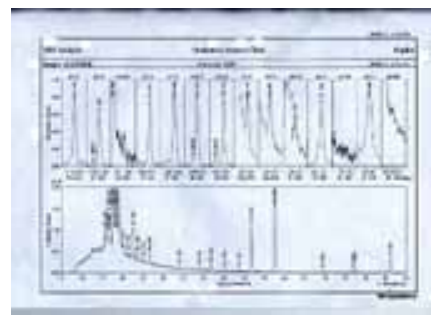

Figure 2. Experimental Result (A) 


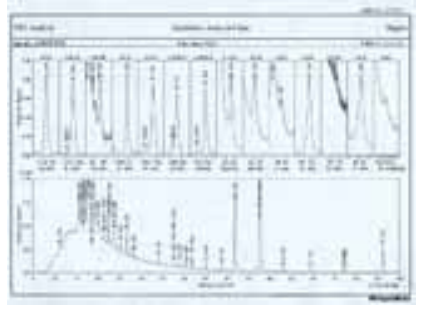

Figure 3. Experimental Result (B)

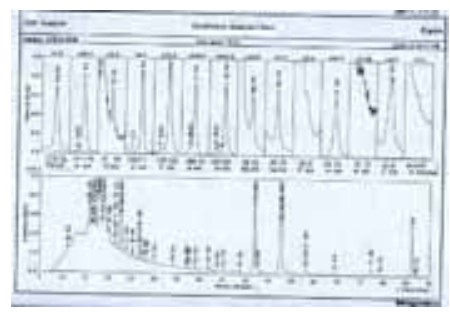

Figure 4. Experimental Result (C)

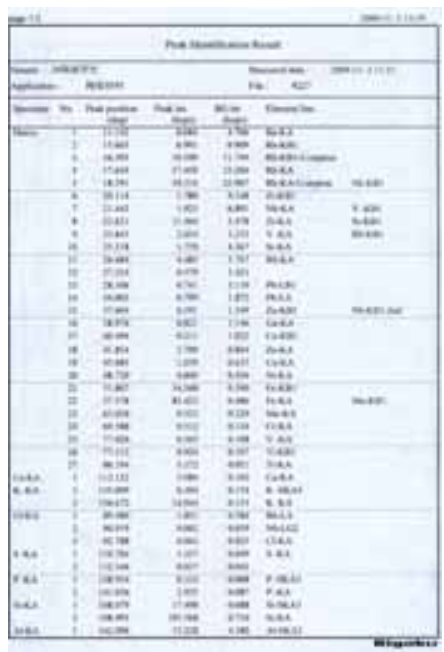

(a)

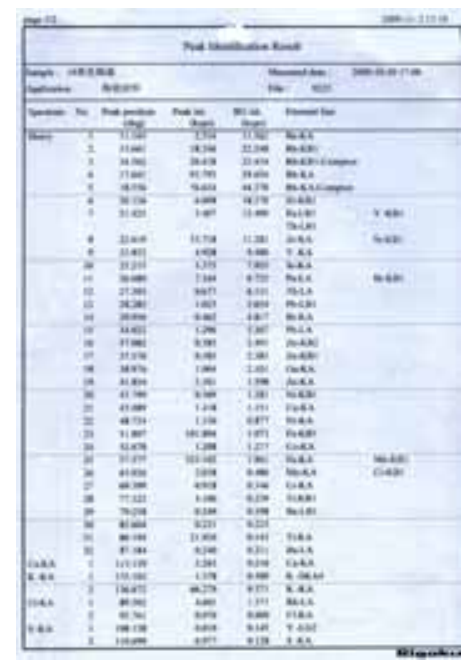

(b)

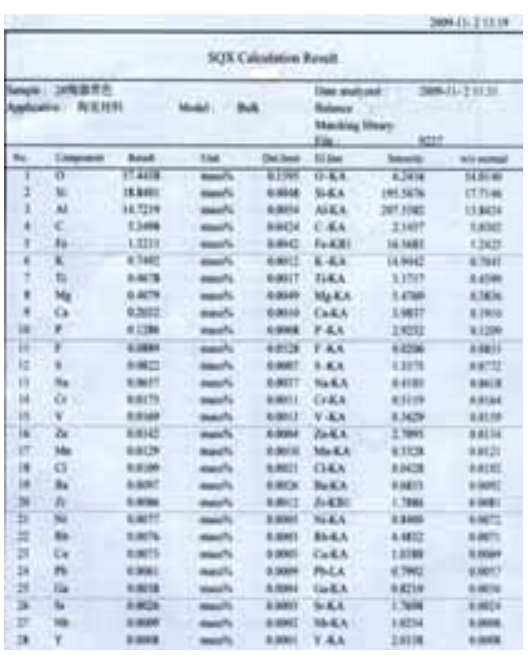

(c)

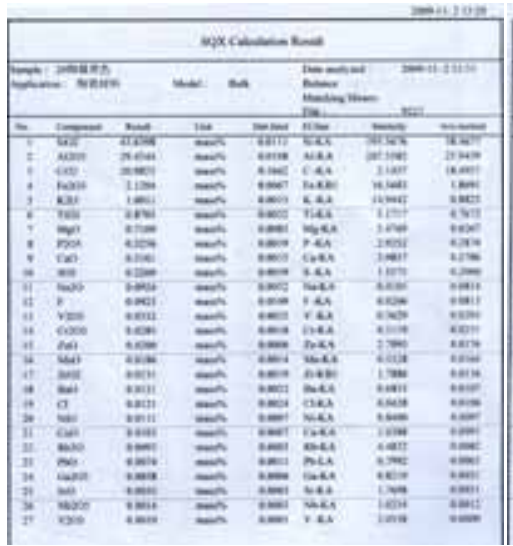

(d)

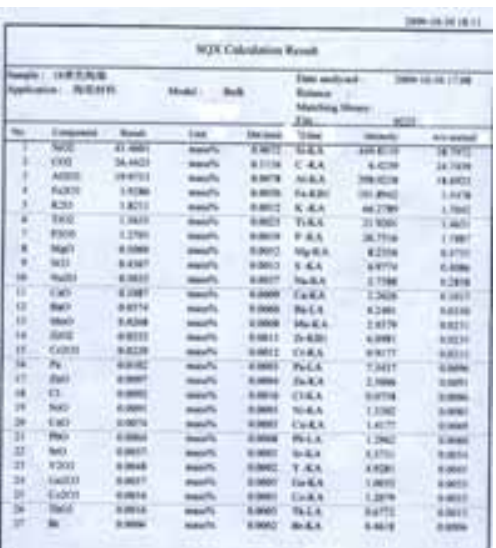

(e)

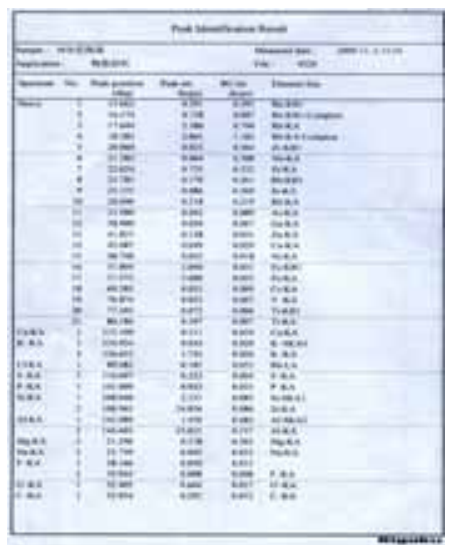

(f) 


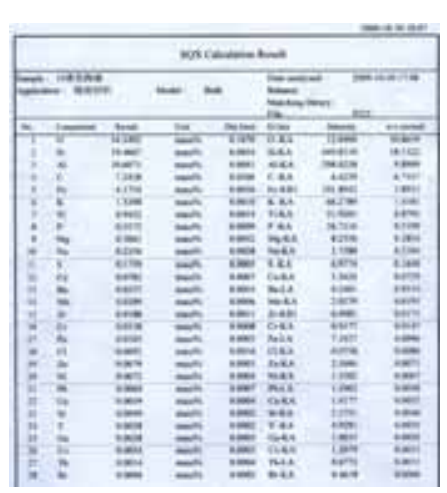

(g)

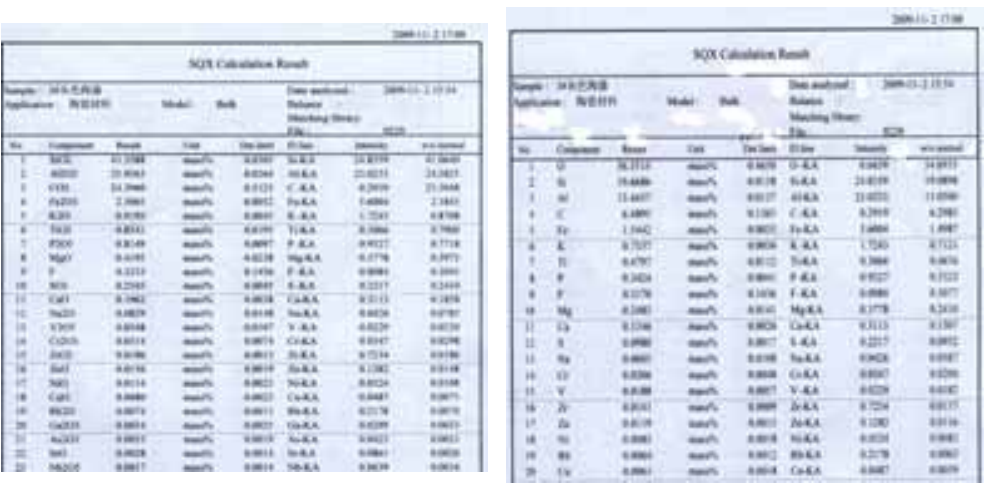

(h) (i)

Figure 5. Experimental Datas 